\title{
AN IMPROVED UPPER BOUND FOR GLOBAL DIMENSION OF SEMIGROUP ALGEBRAS
}

\author{
WILLIAM R. NICO ${ }^{1}$
}

\begin{abstract}
An upper bound for the global dimension of the semigroup algebra of a finite regular monoid in terms of an ideal series for the monoid is determined by the partially ordered set of $J$-classes of the monoid. In particular, if the monoid is combinatorial, the global dimension of the algebra is bounded by the sum of the global dimension of the coefficient ring and twice the length of the longest chain of $\mathscr{J}$-classes in the monoid.
\end{abstract}

In [3] we considered a finite regular monoid $S$ and a composition series of ideals $S=I_{1} \supset I_{2} \supset \cdots \supset I_{n}$. For a commutative ring with identity $k$ we then defined $\sigma\left(I_{j}\right)=0,1$, or 2 , respectively, depending on whether the algebra $k I_{j} / k I_{j+1}$ has a two-sided identity, has no two-sided identity but has a right or left identity, or has neither right nor left identity. Then we set $\mu(S)=\sigma\left(I_{1}\right)+\sigma\left(I_{2}\right)+\cdots+\sigma\left(I_{n}\right)$ and found an upper bound for l.gl.dim $k S$ which involved $\mu(S)$. In particular, if $S$ is combinatorial (i.e., each subgroup is trivial), or if $k G$ is semisimple for

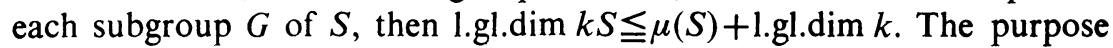
of this paper is to show that an estimate $\tau(S)$ can be computed as above using an ideal series for $S$ which is in general shorter than a composition series. For semigroup terminology we follow [1] or [2].

We form an ideal series $S=I_{1}^{\prime} \supset I_{2}^{\prime} \supset \cdots \supset I_{m}^{\prime}$ as follows: Let $I_{m}^{\prime}$ be the unique minimal ideal of $S$, and for $r=m-1, \cdots, 1$ let $I_{r}^{\prime}$ be such that $I_{r}^{\prime} \mid I_{r+1}^{\prime}$ is the union of all 0 -minimal ideals in $S / I_{r+1}^{\prime}$. In this ideal series $m$ is the length of the longest chain in the partially ordered set of $\mathscr{J}$-classes of $S$. In particular, $m=n$ if and only if $S$ is a chain of $\mathscr{J}$-classes; otherwise $m<n$.

Define $\sigma\left(I_{j}^{\prime}\right)$ in terms ol i,entities of $k I_{j}^{\prime} / k I_{j+1}^{\prime}$ as was done for the ideals in the composition series above. Let $\tau(S)=\sigma\left(I_{1}^{\prime}\right)+\cdots+\sigma\left(I_{m}^{\prime}\right)$. Then

Received by the editors November 15, 1971.

AMS 1970 subject classifications. Primary 16A60, 20M25; Secondary 18G20, $18 \mathrm{G} 15$.

Key words and phrases. Homological dimension, semigroup, semigroup algebra, rings.

${ }^{1}$ Supported in part by National Science Foundation grant GP-29437. 
we have

THEOREM. Let $S$ be a finite regular monoid and $k$ be a commutative ring with identity. If $S$ is combinatorial or if $k G$ is semisimple for each subgroup $G$ of $S$, then l.gl.dim $k S \leqq \tau(S)+1 . g l . d i m ~ k$.

The proof is by induction and the following lemma.

LEMMA. Let $S$ be a regular monoid with zero, and let $I$ be the union of the 0-minimal ideals of $S$. Then $I=J_{1}^{0} \cup \cdots \cup J_{p}^{0}$ where each $J_{i}$ is a regular $\mathscr{J}$-class of $S$ with maximal subgroup $G_{i}$. Let $T=S / I, k$ be a commutative ring with identity, and $k_{0} S$ be the contracted semigroup algebra of $S$. If for any left $k_{0} S$-module $M$ w'e let $d_{k_{0} S}(M)$ be the projective dimension of $M$, then

$$
d_{k_{0} S}(M) \leqq \max \left\{\max \left\{1 . g l \cdot \operatorname{dim} k G_{i}: i=1, \cdots, p\right\}, \lg \operatorname{ld} \cdot \operatorname{dim} k_{0} T+\sigma(I)\right\} .
$$

Proof. Observe $k_{0} T=k_{0} S / k_{0} I$. As in [3] we see that $d_{k_{0} S}\left(k_{0} T\right)=0$, 1 resp., depending on whether $k_{0} I$ has or lacks a right identity.

Letting $M^{*}=M /\left(k_{0} I\right) M$, we see that $M^{*}$ is a $k_{0} T$-module and that $d_{k_{0} S}\left(M^{*}\right) \leqq d_{k_{0} T}\left(M^{*}\right)+d_{k_{0} S}\left(k_{0} T\right) \leqq$ l.gl.dim $k_{0} T+d_{k_{0} S}\left(k_{0} T\right)$ by a standard result.

We observe that $k_{0} I=k_{0} J_{1} \oplus k_{0} J_{2} \oplus \cdots \oplus k_{0} J_{p}$, so that $\left(k_{0} I\right) M=$ $\left(k_{0} J_{1}\right) M+\cdots+\left(k_{0} J_{p}\right) M$. Now if $e_{i}$ is the identity of $G_{i}$, it is known that $J_{i}^{0}=S e_{i} S$. We may thus define $M^{* *}$ by the exact sequence

$$
0 \rightarrow M^{* *} \rightarrow \bigoplus_{i=1}^{p}\left(k_{0} S e_{i} \otimes_{k G_{i}} e_{i} M\right) \rightarrow\left(k_{0} I\right) M \rightarrow 0,
$$

where the right hand map is given by $\left(x_{1} \otimes m_{1}, \cdots, x_{p} \otimes m_{p}\right) \mapsto x_{1} m_{1}+\cdots+$ $x_{p} m_{p}$. Now if $\left(u_{1}, \cdots, u_{p}\right) \in M^{* *}$ and $x \in J_{1}$, then $x\left(u_{1}, \cdots, u_{p}\right)=$ $\left(x u_{1}, 0, \cdots, 0\right)$ since $J_{1}$ annihilates $J_{i}, i=2, \cdots, p$. Now the argument of [3] shows that $x_{1} u_{1}=0$ also; hence we show that $M^{* *}$ is a $k_{0} T$-module and that if $k_{0} I$ has a left identity, $M^{* *}=0$.

Thus we have $d_{k_{0} S}\left(M^{* *}\right) \leqq 1 . g l \cdot d i m k_{0} T+d_{k_{0} S}\left(k_{0} T\right)$. By [3],

$$
d_{k_{0} S}\left(k_{0} S e_{i} \otimes_{k G_{i}} e_{i} M\right)=d_{k G_{i}}\left(e_{i} M\right) \text {. }
$$

By standard results we have

and

$$
d_{k_{0} S}(M) \leqq \max \left\{d_{k_{0} S}\left(k_{0} I M\right), d_{k_{0} S}\left(M^{*}\right)\right\}
$$

$d_{k_{0} S}\left(k_{0} I M\right) \leqq \max \left\{d_{k_{0} S}\left(M^{* *}\right), \max \left\{d_{k_{0} S}\left(k_{0} S e_{i} \otimes_{k G_{i}} e_{i} M\right): i=1, \cdots, p\right\}\right\}$.

These inequalities establish the lemma. 


\section{REFERENCES}

1. M. A. Arbib (Editor) Algebraic theory of machines, languages, and semigroups, Academic Press, New York, 1968. MR 38 \#1198.

2. A. H. Clifford and G. B. Preston, The algebraic theory of semigroups. Vol. 1, Math. Surveys, no. 7, Amer. Math. Soc., Providence, R.I., 1961. MR 24 \#A2627.

3. W. Nico, Homological dimension in semigroup algebras, J. Algebra 18 (1971), 404-413. MR 43 \#3359.

Department of Mathematics, Tulane University, New Orleans, Louisiana 70118 